Alberta Thy-32-92

UMN-TH-1109/92

December 1992

\title{
Physical Properties of Four Dimensional Superstring Gravity Black Hole Solutions
}

\author{
Bruce A. Campbell and Nemanja Kaloper \\ Department of Physics, University of Alberta \\ Edmonton, Alberta, Canada T6G 2J1 \\ Richard Madden and Keith A. Olive \\ School of Physics and Astronomy, University of Minnesota \\ Minneapolis, Minnesota, 55455 USA
}

\begin{abstract}
We consider the physical properties of four dimensional black hole solutions to the effective action describing the low energy dynamics of the gravitational sector of heterotic superstring theory. We compare the properties of the external field strengths in the perturbative solution to the full $O\left(\alpha^{\prime}\right)$ string effective action equations, to those of exact solutions in a truncated action for charged black holes, and to the Kerr-Newman family of solutions of Einstein-Maxwell theory. We contrast the numerical results obtained in these approaches, and discuss limitations of the analyses. Finally we discuss how the new features of classical string gravity affect the standard tests of general relativity.
\end{abstract}




\section{Introduction}

Superstrings [1] have emerged as candidates to provide a unified, quantum, description of matter, gauge forces, and spacetime geometry. As prospective "theories of everything" they are economical in their fundamental principles. At the present (first quantized) level of description, a string theory is specified by the fields (coordinate and other) carried by the string world sheet. For consistent first quantized propagation of a (closed) string, the quantum theory of the string world-sheet must satisfy reparametrization and Weyl invariance, and the particular type of string (bosonic, heterotic, type II) is determined by the number of local chiral supersymmetries relating the world sheet fields. In a (super)conformal gauge this requires that the world-sheet theory be a modular invariant conformal field theory with total central charge (including gauge-fixing ghosts) equal to zero. World sheet theories satisfying these conditions then define consistent string propagation, and from the point of view of second-quantized string theory would correspond to propagation in different classical solutions of the second quantized theory.

So the taxonomy of the string world sheet reflects the multiplicity of classical solutions of the second-quantized theory. In this view, the number of dimensions of spacetime, the gauge group, the fermion families, et cetera, reflect the properties of a particular solution of the underlying theory of second-quantized string. At the present time literally millions of constructions for consistent string world-sheet theories are known, and we have at present no method of classifying all solutions, or even of knowing if such a classification exists. In the absence of a classification of string solutions, we will only be able to extract predictions from, and make tests of, string theory, if we can find general features of the plethora of string solutions which must hold in any solution purporting to describe our universe.

In fact, there is one universal feature shared by critical strings propagating in spacetimes which are large compared to the characteristic string size (strictly speaking, from the world-sheet point of 
view we should say strings with world-sheet fields which include coordinate fields parametrizing a manifold which is regular and large in string units). This is the presence in the spectrum of closed strings of a tensor excitation, the metric (graviton); the dynamics of this excitation computed from correlation functions of its vertex operator reduce at long wavelengths (on the coordinate manifold) to the dynamics of Einstein gravity (plus higher order corrections) on the manifold, with this mode as the Einstein metric. In short, any critical string theory solution with a large spacetime, predicts gravitation in that spacetime. Historically it was this unexpected result that elevated string theory to candidacy for a unified theory of all the interactions. In the present context, the existence of gravitation in all string solutions with large spacetimes, opens the prospect of direct tests of string theory by investigation of the nature of string gravity, which is an inescapable consequence of the theory.

Since it is the gravitational sector for which string theory gives construction (solution) independent features, to test it one should consider systems whose structure is essentially determined by gravitation. The unique class of objects for which this is known to be true are the black holes that arise in the theory. This is precisely the reason for the extensive studies of the black holes in string theory conducted recently, where they have been investigated with varying degree of approximation [4] - [23]. It is the purpose of this paper to analyze the structure and physical properties of the classical black hole solutions that arise in string gravity. We include the complete set of next order corrections in $\alpha^{\prime}$ (the inverse string tension) in the string gravity effective Lagrangian, and examine the modifications that they produce to black hole solutions both analytically and numerically. We will compare our results to those that have been obtained when only a subset of the corrections are included. We also examine how our solutions, considered as approximate exterior solutions to astrophysical bodies, would modify the standard experimental tests of relativistic gravity.

In closed, oriented, critical, strings, the graviton excitation is part of the gravitational multiplet of (classically) massless particles. It includes the symmetric tensor graviton $g_{M N}$, a scalar dilaton 
$\phi$, and an antisymmetric tensor two-form field $B_{M N}$. The effective action for the leading order (independent of $\alpha^{\prime}$ ) gravitational dynamics of the long wavelength modes (in the Einstein conformal frame) consists of the the Einstein action for the graviton, a dilaton kinetic term, and a dilaton rescaled kinetic term for the Kalb-Ramond form field. At the next order $O\left(\alpha^{\prime}\right)$ in the expansion of the effective action for the heterotic string, there appear gauge field kinetic terms for any gauge groups appearing in the string construction, as well as higher order gravitational curvature terms, and Chern-Simons modifications of the Kalb-Ramond field (plus of course construction dependent matter sector fields which are not of interest to us). To $O\left(\alpha^{\prime}\right)$ the effective Lagrangian describing the dynamics of the modes of interest to us may be written [2, 3]:

$$
S=\int d^{4} x \sqrt{g}\left\{\frac{R}{2 \kappa^{2}}-6 e^{-2 \sqrt{2} \kappa \phi} H^{\mu \nu \lambda} H_{\mu \nu \lambda}-\frac{1}{2} \partial_{\mu} \phi \partial^{\mu} \phi+\frac{\alpha^{\prime}}{16 \kappa^{2}} e^{-\sqrt{2} \kappa \phi}\left(\hat{R}^{2}-F_{\mu \nu} F^{\mu \nu}\right)\right\}
$$

where the effective action (1) has been expanded in the string tension to $O\left(\alpha^{\prime}\right) . H_{\mu \nu \lambda}$ are the components of the three-form

$$
H_{\mu \nu \lambda}=\partial_{[\lambda} B_{\mu \nu]}+\frac{\alpha^{\prime}}{8 \kappa}\left(\Omega_{3 L_{\mu \nu \lambda}}-\Omega_{3 Y_{\mu \nu \lambda}}\right)
$$

where $B_{\mu \nu}$ is the antisymmetric field in the gravitational multiplet, and the gauge, and Lorentz, Chern-Simons terms are given by:

$$
\begin{aligned}
\Omega_{3 Y_{\mu \nu \lambda}} & =\frac{1}{2} \operatorname{Tr}\left(A_{[\lambda} F_{\mu \nu]}-\frac{2}{3} A_{[\mu} A_{[\nu} A_{\lambda]]}\right) \\
\Omega_{3 L_{\mu \nu \lambda}} & =\frac{1}{2} \operatorname{Tr}\left(\omega_{[\lambda} R_{\mu \nu]}-\frac{2}{3} \omega_{[\mu} \omega_{[\nu} \omega_{\lambda]]}\right)
\end{aligned}
$$

where the trace is taken over the (suppressed) gauge and Lorentz indices, and the spin connection $\omega_{\mu}^{a b}=-e^{b \alpha} e_{\alpha ; \mu}^{a}$ with vierbein $e_{\mu}^{a}$. Note that the $H^{2}$ term in (1) already contains terms of $O\left(\alpha^{\prime}\right)$. In the action (11) $\phi$ is the dilaton and the Gauss-Bonnet combination is given by $\hat{R}^{2}=R_{a b c d} R^{a b c d}-$ $4 R_{a b} R^{a b}+R^{2}$. 
It is useful to dualize the Kalb-Ramond field in order to express the action in terms of dynamical modes. Let us re-express the action in terms of the dual of $H, Y={ }^{*} H$.

$$
Y_{\sigma}=\sqrt{g} \epsilon_{\mu \nu \rho \sigma} H^{\mu \nu \rho}
$$

from equation (2), we have the Bianchi identity

$$
d H=\frac{\alpha^{\prime}}{8 \kappa} \operatorname{Tr}(R \wedge R-F \wedge F)
$$

which we can write as

$$
\left(\sqrt{g} Y^{\rho}\right)_{, \rho}=\frac{\alpha^{\prime}}{8 \kappa} \frac{\epsilon^{\mu \nu \lambda \sigma}}{4}\left(R_{a b \mu \nu} R_{\lambda \sigma}^{b a}+F_{\mu \nu} F_{\lambda \sigma}\right)
$$

Using the constraint (7), we can write the action in terms of the truly dynamical axion mode of the theory as

$$
\begin{aligned}
S=\int d^{4} x \sqrt{g}\left\{\frac{R}{2 \kappa^{2}}\right. & -\frac{1}{144} e^{2 \sqrt{2} \kappa \phi} \partial_{\mu} b \partial^{\mu} b-\frac{1}{2} \partial_{\mu} \phi \partial^{\mu} \phi \\
& \left.+\frac{\alpha^{\prime}}{16 \kappa^{2}} e^{-\sqrt{2} \kappa \phi}\left(\hat{R}^{2}-F_{\mu \nu} F^{\mu \nu}\right)-b \frac{\alpha^{\prime}}{8 \kappa} \frac{\epsilon^{\mu \nu \rho \lambda}}{4 ! \sqrt{g}}\left(R_{a b \mu \nu} R_{\rho \lambda} b a+F_{\mu \nu} F_{\rho \lambda}\right)\right\}
\end{aligned}
$$

where $Y^{\rho}=\frac{1}{12} \exp (2 \sqrt{2} \kappa \phi)\left(\partial^{\rho} b\right)$. If one neglects the space-time dependence of the dilaton (consistently in the perturbative expansion when dilaton dependence occurs at $O\left(\alpha^{\prime}\right)$ ), this leads to the axion equation of motion

$$
\square a=\frac{6 \alpha^{\prime}}{8 \kappa} \frac{\sqrt{2} \epsilon^{\mu \nu \rho \lambda}}{4 ! \sqrt{g}}\left(R_{a b \mu \nu} R_{\rho \lambda}^{b a}+F_{\mu \nu} F_{\rho \lambda}\right)
$$

where $a$ is the rescaled axion which approximates the one with canonical kinetic term, $\partial^{\rho} a=$ $\exp (2 \sqrt{2} \kappa \phi) \partial^{\rho} b / 6 \sqrt{2}$. [Because we are anticipating that the dilaton $\phi \sim \alpha^{\prime}$, we neglect the exponentials in writing equation (9)]. The dilaton equation of motion is now rather easily obtained from the action (8), and the axion field redefinition

$$
\square \phi=\frac{\alpha^{\prime} \sqrt{2}}{16 \kappa} \exp (-\sqrt{2} \kappa \phi)\left(\hat{R}^{2}-F_{\mu \nu} F^{\mu \nu}\right)+\sqrt{2} \kappa \exp (-2 \sqrt{2} \kappa \phi)\left(\partial_{\mu} a\right)\left(\partial^{\mu} a\right)
$$


Again when the solution for $a$ is already of order $\alpha^{\prime}$, we may drop the last term (consistently in our expansion); expanding the exponential in the dilaton as before, and keeping terms of order $\alpha^{\prime}$, we arrive at

$$
\square \phi=\frac{\alpha^{\prime} \sqrt{2}}{16 \kappa}\left(\hat{R}^{2}-F_{\mu \nu} F^{\mu \nu}\right)
$$

Note that both the axion and the dilaton are first non-trivial at order $\alpha^{\prime}$.

In [5, 6, 77, 10] we have evaluated the source terms for the axion and dilaton for the background of a weakly-charged, rotating black hole. We convoluted these sources with the Green function for that background, to compute the classical hair produced in the Kerr-Newman background. We kept all effects to $O\left(\alpha^{\prime}\right)$ perturbatively for the slowly-rotating, weakly-charged case. To the order in which we worked, back-reaction on the metric could be neglected, and it remains of Kerr-Newman form [see appendix]. The explicit forms of the dilaton and axion hair we obtained are also shown in the appendix. It is our purpose in this paper to compare our perturbative solution to exact solutions of truncations of our action, both perturbatively and numerically, as well as to discuss the physically measurable effects of the dilaton and axion hair produced.

\section{Black Holes And String Theory}

In order to elaborate on the difference between the classical General Relativity Kerr-Newmann family of black hole solutions (KN), our $\alpha^{\prime}$ string dilaton/axion hair solutions [10 (hereafter CKO) and the dilaton/axion solutions by Gibbons [11], Gibbons and Maeda [12, Garfinkle, Horowitz and Strominger [13], Shapere, Trivedi and Wilczek [14 and Sen [19] (which hereafter we refer to as GS), it will be useful to first describe how these solutions are similar. For simplicity, we will consider the non-rotating case. The similarities and differences are not qualitatively affected when $A \neq 0$. Recall that the solutions of GS were found by beginning with the string action (1) in the $\alpha^{\prime}$ expansion, truncating the action to $O\left(\alpha^{\prime}\right)$ but keeping only one of the many $O\left(\alpha^{\prime}\right)$ contributions, namely the 
gauge kinetic term. With this truncation, they find the remarkable closed form solution given in the appendix.

To compare the GS solution (with $A=0$ ) for the metric and the standard RN solution we need to perform a coordinate transformation:

$$
r^{2}=\rho^{2}-\frac{Q^{\prime 2} \rho}{G M}=\tilde{\rho}^{2}+\frac{\tilde{Q}^{2} \tilde{\rho}}{G M}
$$

This brings the angular part of the metric (the coefficient of $d \Omega$ ) to the standard form. Solving for $\rho(r)$ and $\tilde{\rho}(r)$ and keeping only terms of $O\left(\alpha^{\prime}\right)$ (note that $Q^{\prime 2}=\tilde{Q}^{2}=\alpha^{\prime} Q^{2} / 8$ as described in the appendix) one sees immediately that both metrics (A10) and (A11) reduce exactly to the Reisnner-Nordstrom metric in (A8). This should not be a surprise since it has been shown that the Kerr-Newmann family is the $O\left(\alpha^{\prime}\right)$-expanded metric solution in string theory [10].

In this sense, the GS solution is in fact equivalent to the $\mathrm{KN}$ solution up to an $O\left(\alpha^{\prime}\right)$ coordinate transformation, and hence equivalent to the metric in the CKO solution. Similarly one can see that the dilaton and axion solutions are also equivalent to $O\left(\alpha^{\prime}\right)$. For example, the dilaton solution of GS is

$$
\tilde{\phi}=-\log \left(1+\frac{\tilde{Q}^{2}}{G M \tilde{\rho}}\right) \approx-\frac{\alpha^{\prime} Q^{2}}{8 G M r}
$$

which corresponds to (A12) given in the appendix.

However there are several important aspects to which these solutions differ. First we compare the horizons and critical limits of these solutions (at least formally) to perform the $\alpha^{\prime}$ expansion with large $q^{2}$, in this case $Q^{2}$ must be very large. In the $\mathrm{RN}$ solution, the outer horizon occurs at the radial coordinate value

$$
r_{H}=G M+\sqrt{G^{2} M^{2}-G q^{2}}
$$

and the critical limit (for which there is a naked singularity) is obtained as

$$
\alpha^{\prime} \frac{Q^{2}}{8}=G q^{2}=G^{2} M^{2}=Q^{\prime 2}
$$


In the $\alpha^{\prime}$ expansion of string theory, Eq. (14) can be expanded so that

$$
r_{H}=2 G M-\frac{q^{2}}{2 M}=2 G M-\alpha^{\prime} \frac{Q^{2}}{16 G M}=2 G M-\frac{Q^{\prime 2}}{2 G M}
$$

Otherwise, it is no longer possible to obtain the critical limit from this expression. In the G-S solution the horizon occurs at

$$
\rho_{H}=2 G M
$$

which when related to $r$ by the coordinate transformation Eq. (12) becomes

$$
\rho_{H}=r_{H}+\frac{Q^{\prime 2}}{2 G M}=2 G M
$$

so that $r_{H}$ is given precisely by (16). (In terms of $\tilde{\rho}, \tilde{\rho}_{H}=2 G M-\tilde{Q}^{2} / M$ with $A=0$.) The horizons in the two solutions are again equivalent in $\alpha^{\prime}$ expansion up to a coordinate transformation. The critical limit is however different and now occurs when

$$
Q^{\prime 2}=2 G^{2} M^{2}
$$

Note that despite $Q^{\prime 2} \propto \alpha^{\prime}, Q^{\prime 2}$ can be thought of as large, so long as $Q^{2}$ is large, i.e. one can work in the limit of small $\alpha^{\prime}$ and fixed $Q^{\prime 2}$. Similarly, the radii at which the surface area goes to zero are also the same for small $\alpha^{\prime} Q^{2}$. In the GS solution

$$
A=4 \pi \rho\left(\rho-\frac{Q^{\prime 2}}{G M}\right)
$$

the area vanishes at the hypersurface corresponding to $\rho=Q^{\prime 2} / G M$. Under the coordinate transformation (12),

$$
A=4 \pi r^{2}
$$

as in the RN solution.

The temperature of the black holes also agree at small $Q^{\prime 2}$ while they differ at large $Q^{\prime 2}$. Writing $T=K / 2 \pi$ with

$$
K=\frac{1}{2}\left(\frac{\partial g_{00}}{\partial r}\right)_{r=r_{H}}
$$


In the GS solution

$$
K=\frac{1}{2}\left(\frac{2 G M}{\rho_{H}^{2}}\right)=\frac{1}{4 G M}
$$

For the RN solution,

$$
K=\frac{\sqrt{G^{2} M^{2}-G q^{2}}}{G^{2} M^{2}\left(1+\sqrt{1-\frac{q^{2}}{G M^{2}}}\right)^{2}} \approx \frac{1}{4 G M}\left(1-\frac{q^{2}}{2 G M^{2}}\right)\left(1+\frac{q^{2}}{2 G M^{2}}\right) \approx \frac{1}{4 G M}
$$

as in (23). In the small $Q^{\prime 2}$ limit, these expressions for $\mathrm{T}$ are indistinguishable, while for large $Q^{\prime 2}$, the difference is clearly manifest.

In the large $Q^{\prime 2}$ limit, the coordinate transformations (16) are no longer valid, and the solutions appear different. Thus it is important to better understand this limit to determine whether or not these new solutions are in some sense better stringy black hole solutions. To determine precisely what that limit is, we need to inspect terms in the action which have been discarded. Indeed, it is not obvious that higher order curvature terms such as Gauss-Bonnet and $\alpha^{\prime} R^{3}$ [2, 3, 24] terms are small compared with the terms kept in $(A 1, A 4)$. The reason for possible concern is that as one approaches the critical limit $Q^{\prime 2}=2 G M^{2}$, the curvature scalar blows up on the horizon at $\rho=2 M$,

$$
R=\frac{Q^{\prime 4}(2 G M-\rho)}{2 \rho^{3}\left(Q^{\prime 2}-G M \rho\right)^{2}} \rightarrow \frac{8 G^{2} M^{2}}{\rho^{3}(2 G M-\rho)} \propto \frac{1}{x}
$$

with $x=2 G M-\rho$. Indeed, in the critical limit, other terms in the action also blow up, such as

$$
\partial_{\mu} \phi^{\prime} \partial^{\mu} \phi^{\prime}=\left(\frac{Q^{\prime 2}}{2\left(G M \rho-Q^{\prime 2}\right)}\right)^{2}\left(1-\frac{2 G M}{\rho}\right) \rightarrow \frac{G^{2} M^{2}}{\rho^{3}(\rho-2 G M)} \propto \frac{1}{x}
$$

and

$$
e^{-2 \phi^{\prime}} F^{2}=\frac{Q^{\prime 2}}{\rho^{4}}\left(1-\frac{2 G M}{\rho}\right)^{-1} \rightarrow \frac{2 G^{2} M^{2}}{\rho^{3}(2 G M-\rho)} \propto \frac{1}{x}
$$

(here we give the expression for the electrically charged case, the general case is equally divergent). One might expect a priori, that other $O\left(\alpha^{\prime}\right)$ terms would blow up more strongly (as $x^{-2}$ or worse). Should this occur, the truncation keeping only the $\alpha^{\prime} F^{2} \exp \left(-2 \phi^{\prime}\right)$ term would be invalid except 
at small $Q^{\prime 2}$ where all solutions are equivalent anyway. As we will see, the answer to this question depends heavily on whether or not the black hole posseses any magnetic charge.

First, let us recall the generalization of these solutions when both electric and magnetic charges are present. In the KN solution $q^{2}=q_{e}^{2}+q_{m}^{2}$ and in the CKO solution for the dilaton (and axion) the general solution is given in $(A 12, A 13)$. The GS dilaton solution can be generalized by taking $Q^{\prime 2}=Q_{e}^{\prime 2}+Q_{m}^{\prime 2}$ and $(\llbracket 14)$

$$
e^{-2 \phi^{\prime}}=Q^{\prime 2} \frac{1-\frac{Q^{\prime 2}}{G M \rho}}{Q_{m}^{\prime 2}+Q_{e}^{\prime 2}\left(1-\frac{Q^{\prime 2}}{G M \rho}\right)^{2}}
$$

or in the critical limit

$$
e^{-2 \phi^{\prime}}=\frac{2 G^{2} M^{2} \rho(\rho-2 G M)}{Q_{m}^{\prime 2} \rho^{2}+Q_{e}^{\prime 2}(\rho-2 G M)^{2}}=-\frac{2 G^{2} M^{2} \rho x}{Q_{m}^{\prime 2} \rho^{2}+Q_{e}^{\prime 2} x^{2}}
$$

As one can see, the limiting behavior of $\exp \left(-2 \phi^{\prime}\right)$ as $x \rightarrow 0$ depends crucially on whether or not $Q_{m}^{\prime}=0$.

To test the limit, we examine several additional $O\left(\alpha^{\prime}\right)$ contributions. For example, the GaussBonnet combination (in the critical limit)

$$
\alpha^{\prime} e^{-2 \phi^{\prime}} \hat{R}^{2}=\alpha^{\prime} \frac{16 G^{2} M^{2}(2 G M-3 \rho)}{\rho^{6}(\rho-2 G M)} e^{-2 \phi^{\prime}}=\alpha^{\prime} \frac{32 G^{4} M^{4}}{\rho^{5}} \frac{(2 G M-3 \rho)}{Q_{m}^{\prime 2} \rho^{2}+Q_{e}^{\prime 2} x^{2}}
$$

which for $Q_{m}^{\prime} \neq 0$ is finite as $x \rightarrow 0$ but diverges as $x^{-2}$ if $Q_{m}^{\prime}=0$. There are additional dilaton terms

$$
\alpha^{\prime} e^{-2 \phi^{\prime}}\left(D^{\mu} \partial^{\nu} \phi^{\prime}\right)\left(\partial_{\mu} \phi^{\prime}\right)\left(\partial_{\nu} \phi^{\prime}\right) \rightarrow\left\{\begin{array}{cc}
\alpha^{\prime} \frac{G^{3} M^{3}(3 G M-2 \rho)}{\rho^{7} x} & \left(Q_{e}=0\right) \\
-\alpha^{\prime} \frac{G^{3} M^{3}(3 G M-2 \rho)}{\rho^{5} x^{3}} & \left(Q_{m}=0\right)
\end{array}\right.
$$

and

$$
\alpha^{\prime} e^{-2 \phi^{\prime}}\left(\left(\partial_{\mu} \phi^{\prime}\right)\left(\partial^{\mu} \phi^{\prime}\right)\right)^{2} \rightarrow \begin{cases}-\alpha^{\prime} \frac{G^{4} M^{4}}{\rho^{7} x} & \left(Q_{e}=0\right) \\ -\alpha^{\prime} \frac{G^{4} M^{4}}{\rho^{5} x^{3}} & \left(Q_{m}=0\right)\end{cases}
$$

Although the $Q_{e}=0$ contributions diverge as $x^{-1}$ (as do the terms included in $(A 1, A 4)$ ), they are suppresed by a factor $\alpha^{\prime} G^{2} M^{2} / \rho^{4} \propto \alpha^{\prime} / G^{2} M^{2} \propto 1 / G M^{2}$. Then for massive black holes, $G M^{2}>>1$, even these divergent terms can be safely neglected. However for $Q_{m}=0$, these terms diverge as 
$x^{-3}$ and can not be neglected when studying the critical behavior or the solution near the horizon. Similarly other terms in the action to order $\alpha^{\prime}$ involving only $\phi^{\prime}, R$ or $F$ show the same behavior. They diverge as $x^{-1}$ but are suppresed by $1 / G M^{2}$ when $Q_{m} \neq 0$ and diverge as $x^{-3}$ when $Q_{m}=0$. We also check the model independent curvature cubed term at $O\left(\alpha^{\prime 2}\right)$, 24

$$
\alpha^{\prime 2} e^{-4 \phi^{\prime}}\left(3 I_{1}-4 I_{2}\right) \rightarrow 48 G^{3} M^{3} \alpha^{2} \frac{13 G^{3} M^{3}-12 G^{2} M^{2} \rho+4 G M \rho^{2}-2 \rho^{3}}{\rho^{11}} \begin{cases}\frac{1}{x} & \left(Q_{e}=0\right) \\ \frac{\rho^{4}}{x^{5}} & \left(Q_{m}=0\right)\end{cases}
$$

which again only diverges as $x^{-1}$, and is suppresed as $\alpha^{2} / G^{4} M^{4} \propto 1 / G^{2} M^{4}$ when $Q_{e}=0$ but severely diverges as $x^{-5}$ when $Q_{m}=0$. The curvature cubed invariants $I_{1}, I_{2}$ are defined by 24

$$
\begin{aligned}
& I_{1}=R_{\mu \nu}{ }^{\alpha \beta} R_{\alpha \beta}{ }^{\gamma \sigma} R_{\gamma \sigma}{ }^{\mu \nu} \\
& I_{2}=R_{\mu \nu}{ }^{\alpha \beta} R_{\nu}{ }^{\gamma \beta \sigma} R_{\gamma \mu \sigma}{ }^{\alpha}
\end{aligned}
$$

To understand the pathological behavior of the solutions in the critical limit we can restrict our attention to purely electric or magnetic solutions. Then, as was described in [13], the gauge fields in the two cases are related by a duality transformation involving the dilaton, itself transforming according to $\phi \rightarrow-\phi$. It is this sign difference which is responsible for the stability of the magnetically charged critical solutions under $O\left(\alpha^{\prime}\right)$ corrections. Indeed, in this case we can conformally transform the metric to the world-sheet frame according to $g_{\mu \nu}=\exp (-\sqrt{2} \kappa \phi) G_{\mu \nu}$. The metric takes the form

$$
d s_{W S}^{2}=d t^{2}-\left(1-\frac{2 G M}{\rho}\right)^{-2} d \rho^{2}-\rho^{2} d \Omega
$$

and as Garfinkle et al. [13] show is non-singular. In fact it corresponds to the geometry of an infinite throat, with the singularity removed to (unreachable) spatial negative infinity. As such, higher order curvature corrections in the $\alpha^{\prime}$ expansion may be expected to be benign for large black holes, which have large "diameter" throat. Since the world-sheet action then takes the form

$$
S=\int d^{4} x \sqrt{G} e^{-\sqrt{2} \kappa \phi}\left\{\frac{R_{W S}}{2 \kappa^{2}}-6 H^{\mu \nu \lambda} H_{\mu \nu \lambda}+\partial_{\mu} \phi \partial^{\mu} \phi+\frac{\alpha^{\prime}}{16 \kappa^{2}}\left(\hat{R}_{W S}^{2}-F_{\mu \nu} F^{\mu \nu}\right)+\ldots\right\}
$$


and all the terms in the parenthesis behave very softly inside the throat (the most singular terms being the dilaton contributions), we see that the worst singular behavior originates from the effective coupling $\exp (-\sqrt{2} \kappa \phi)$ and its variation. In the electrically charged critical solution the situation is reversed due to the fact that the dilaton solution is the inverse of the magnetically charged one and hence the picture of an infinite throat world-sheet geometry is destroyed, as conformal rescaling makes singularities harder. Thence the difference between the $Q_{e}=0$ and $Q_{m}=0$ cases. We should note, however, that although the magnetically charged critical solution is stable under $O\left(\alpha^{\prime}\right)$ (and possibly higher) corrections, it is subject to large quantum corrections; as is well known, the quantum corrections in string theory correspond to higher genus world-sheets, involving powers of the effective coupling $\exp (-\sqrt{2} \kappa \phi)$ (the loop counting expansion parameter) in the effective action. Hence going deeper into the throat in the magnetically charged critical case would make these contributions more significant, eventually threatening to take us outside of the perturbative regime of the theory [14].

We note that the rotating solution of Sen is free of the pathology (the appearance of corrections which are more singular than the terms in the original $O\left(\alpha^{\prime}\right)$ action) discussed above due to the fact that the presence of the angular momentum regularizes the solution in the critical case, much the same way as the electric-magnetic solutions of $N=4$ supergravity with two independent $U(1)$ gauge field charges [20] - [23]. We recall that the horizon of Sen's solution is $\tilde{\rho}_{H}=G M-\tilde{Q}^{2} / 2 G M+$ $\sqrt{\left(G M-\tilde{Q}^{2} / 2 G M\right)^{2}-A^{2}}$, so the critical limit occurs when $G M-\tilde{Q}^{2} / 2 G M=|A|$, implying that $\tilde{\rho}_{H}=|A|>0$ when $A \neq 0$, whereas the singularity is located at $\tilde{\rho}_{S}=0$ irregardless of the value of angular momentum. The nonrotating GS solution then appears as a special case of the $N=4$ supergravity class. Observation of critical solutions in these two cases demonstrates that even in the critical limit the singularity and the horizon remain separated, except in the special case when the solutions reduce back to GS, as can be seen from the fact that the area of the critical horizons remains nonzero. We should note that in the context of supergravity and superstring models a 
non-renormalization theorem has been proved [20], [21] stating that the classical action for critical solutions is not changed by quantum fluctuations derived from its path integral. The crucial point in the proof is the presence of unbroken supersymmetries in the critical backgrounds. However, in these cases metric in the world-sheet conformal frame is not singularity-free and hence the solutions may be sensitive to inclusion of higher order $\alpha^{\prime}$ terms, corresponding to multi-string classical scattering.

We can now conclude the following regarding the difference between the black hole solutions. In the strict $\alpha^{\prime}$ expansion (when $Q^{\prime 2}$ is also small) the GS and CKO solutions are equivalent in all respects up to a coordinate transformation (for $G M^{2} \gg 1$ ). The metric is then also the same as $\mathrm{KN}$, as the backreaction occurs first at $O\left(\alpha^{2}\right)$. When $Q^{\prime 2}$ is large and $Q_{m} \neq 0$, and $G M^{2} \gg 1$, the GS solution offers the best description of the stringy black hole metric and dilaton/axion hair. The truncation used in obtaining the solution is not valid in the critical limit when $Q_{m}=0$. When $Q^{\prime 2}$ is small and $G M^{2} \gtrsim 1$, the CKO solutions for dilaton/axion hair are expected to be better, as the Gauss-Bonnet correction has been included. Finally and unfortunately, when $Q^{\prime 2}$ is large and $G M^{2} \approx 1$, none of these solutions is expected to give an accurate description of the stringy black hole. In the next section, we make some numerical comparisons of the dilaton and axion hair in the two solutions.

\section{Numerical Comparisons Of Solutions}

The axion and dilaton solutions given in Eqs (A12) and (A13) represent expansions (in the angular momentum) of the exact $\left(O\left(\alpha^{\prime}\right)\right)$ integral solutions in terms of Green's functions. The exact equations were integrated numerically and compared (for the monopole, dipole and quadrupole moments) with the expansions given in the appendix. As expected for small values of A, the expansion is quite good. Even for values of $A / G M$ as large as 0.8 , the difference between the exact solution and the expansion was only $10 \%$. 
We now compare the solution of CKO with Sen's exact solution to the truncated action with the coordinate change $r^{2} \rightarrow \tilde{\rho}^{2}+\tilde{Q}^{2} \tilde{\rho} / M$ reducing the angular part of the metric to the standard form. We concentrate on three limiting cases:

1. $G M^{2}=1$ and $Q^{\prime 2} \ll 1$

2. $G M^{2} \gg 1$ and $Q^{\prime 2} / 2 \ll G M^{2}$

3. $G M^{2} \gg 1$ and $Q^{\prime 2} / 2 \approx G M^{2}$

In the fourth logical case, $G M^{2} \approx 1$ and $Q^{\prime 2} / 2 \approx G M^{2}$ neither solution is expected to be good and no comparison was made.

As we indicated in the previous section, in case (1) we expect the solutions to differ because of the inclusion of the Gauss-Bonnet corrections used by CKO. In this regime the CKO solution is more accurate. In Figure 1, where we chose $Q_{e}^{2}=1$ (or $Q^{\prime 2}=1 / 8$ with $\alpha^{\prime}=1$ ) we see the results of this comparison for the axion (1a) and the dilaton (1b). One can see a clear numerical difference for the two solutions due to the importance of the Gauss-Bonnet contributions in this regime. Case (2) is shown in Figure 2. Here we chose $G M^{2}=10^{4}$ and $\left(Q^{\prime 2} / G M^{2}\right)=1.25 \times 10^{-3}$. Our expectation that the solutions are equivalent is clearly borne out.

The final case was a nearly extremal black hole. In this case we expected the GS solution to be better than CKO since we are violating the condition that the charge be small. The results shown in Figure 3 for $G M^{2}=10^{4}$ and $\left(Q^{\prime 2} / G M^{2}\right)=1.53$, unexpectedly show that the solutions remain close even as the extremal limit is approached. However this similarity may be deceptive since the background spacetimes of GS and CKO differ considerably in this limit. In this particular case, the horizon of the GS spacetime is located at an $r$ coordinate value approximately half that of $\mathrm{CKO}$ and the field strength may be continued to much larger values at this smaller $\mathrm{r}$ value. In general, as the extremal limit is approached the horizon of GS retreats to a coordinate value of $r=0$ while 
CKO remains fixed at $r=2 M$ since CKO does not consider the effect of back-reaction of the fields on the metric.

\section{Motion Of Test Particles And Force From Hair}

In this section we will address the question of observing the field hair which we discussed previously. We will attempt to compute measurable effects of the hair upon probes moving in such backgrounds, and analyze deviations from General Relativity that so arise. To gain insight concerning the nature of such effects under the most general circumstances, we will discuss some limiting situations and argue on their basis what happens in more general cases.

We will start by discussing the motion of probes of various structure (made of gravitons, photons, axions, ...) and attempt to determine what is the influence of the hair on them. For simplicity's sake, we will assume as our first case that the black hole background to $O\left(\alpha^{\prime}\right)$ is Schwarzschild, with dilaton hair arising from the Gauss-Bonnet combination. Then a quick glance at the action supports one's naive expectation that the hair should influence motion of different particles differently, for the fields appearing in the action do not take simultaneously canonical form for their kinetic terms. This is due to their coupling to the dilaton, induced on the world-sheet, where all fields couple to it in the same way. Yet we argue that in order to discuss the low energy limit of string theory we should conformally transform the action to the Einstein frame, where the graviton is rendered canonical. Then we can use the notions of standard GR to analyze the solutions since the deviations can be interpreted as a consequence of the extra fields appearing in the theory.

It was argued by Shapere, Trivedi and Wilczek [14] that the motion of quanta of the dilaton and the axion can be determined by conformally rescaling the metric so that in the resulting background the kinetic term of the respective field is canonical and all explicit reference to the dilaton coupling disappears. Then, by analogy with the photon in standard GR, the particles are required to move 
along null geodesics of the conformal transform of the metric. Moreover, as is well known, null geodesics do not change under conformal transformations, and the only difference found in [14] in the motion of different test particles was manifest in the affine parameter measuring intrinsic geometry of a trajectory. As a consequence, it was concluded that the dilaton effects amount to changes in the appearance of the global structure of space-time to different observers as well as variations in local physics, associated with observers in conformally related frames. This argument, however, is not relevant to the motion of photons because the Maxwell Lagrangian density $\sqrt{g} F^{2}$ is a conformal invariant in four dimensions. In other words, no conformal rescaling can yield the canonical kinetic term for the photon. Thus the issue of the motion of photons in a background with hair was left unresolved as ever.

Here we propose an alternative approach to the derivation of the dilaton hair induced force on test particles. We study the geometric optics approximation of a corresponding field equation describing motion of a highly localized solitary wave train. We invoke the validity of the Principle of Equivalence in the Einstein frame, conduct our calculations in a flat Minkowski background, and extend the results to a curved one via the standard minimal prescription, following from the Principle of Equivalence. Our result agrees with the ansätz of [14 in the case of the axion, and extends it to the photon, giving a very similar formula. We then investigate the influence of this, dilaton-induced, force on the motion of test particles.

To start with, we can write down the equations of motion for a generic antisymmetric tensor field, resembling those which can be derived from the low energy action for the axion and the photon. They are the Euler-Lagrange equation,

$$
\partial_{\mu} e^{-\xi \kappa \phi} F^{\mu \nu \lambda \ldots}=0
$$

where $\xi$ is $\sqrt{2}$ for the photon and $2 \sqrt{2}$ for the axion, and the Bianchi identity following from the definition of the field strength as an exact form:

$$
\partial_{[\mu} F_{\nu \lambda \sigma \ldots]}=0
$$


Here we have defined the field strength $p$-form $F_{\mu \nu \lambda \ldots}$ as the exterior derivative of a potential $p$-1-form $A_{\nu \lambda \ldots}, F_{\mu \nu \lambda \ldots}=p ! \partial_{[\mu} A_{\nu \lambda \ldots]}$. Since by its definition $F$ is invariant under $U(1)$ gauge transformations, a gauge has to be fixed for ambiguities to disappear. We choose to work in the Lorenz gauge: $\partial_{\mu} A^{\mu \nu \cdots}=0$. As we are interested in the motion of a solitary wave train, representing a particle, we use for the solution the following ansätz:

$$
A_{\mu \nu \ldots}=f_{a \mu \nu \ldots} e^{\mathrm{i} S}
$$

where $f_{a \mu \nu \ldots}$ is the (antisymmetric) polarization tensor of the particle (wave train), and the phase $S$ is the classical action of the particle (which solves the corresponding Hamilton-Jacobi equation). The index $a$ counts all possible polarization states. The gauge condition then reads $f_{a}{ }^{\mu \nu \cdots} \partial_{\mu} S=0$. Upon substitution of this ansätz in the equations of motion, noting that $\left.F_{\mu \nu \lambda \ldots}=\mathrm{i} p ! \partial_{[\mu} S f_{a} \nu \lambda_{\ldots .}\right]^{\mathrm{i} S}$, we find that the Bianchi identity is satisfied identically (as one expects) and that the Euler-Lagrange equation yields, after canceling the phase $\exp (\mathrm{i} S)$ and separating real and imaginary parts

$$
\begin{gathered}
\partial_{\mu} S \partial^{[\mu} S f_{a}{ }^{\nu \lambda \ldots]}=0 \\
\partial_{\mu}\left(e^{-\xi \kappa \phi} \partial^{[\mu} S f_{a}{ }^{\nu \lambda \ldots]}\right)=0
\end{gathered}
$$

We recall the orthogonality relations of the polarization tensors, $f_{a}{ }^{\mu \nu \cdots} f_{b} \mu \nu \ldots=\delta_{a}$. This at hand, after contracting the equations above with $f_{b} \mu \nu \ldots$ we obtain

$$
\begin{gathered}
(\partial S)^{2} \delta_{a b}-\partial_{\mu} S \partial^{\nu} S f_{a}{ }^{\mu \lambda \ldots} f_{b \nu \lambda \ldots}-(\text { other transpositions })=0 \\
\partial_{\mu}\left(e^{-\xi \kappa \phi} \partial^{\mu} S\right) \delta_{a b}-\partial_{\mu}\left(e^{-\xi \kappa \phi} \partial^{\nu} S\right) f_{a}{ }^{\mu \lambda \ldots} f_{b \nu \lambda \ldots}-(\text { other transpositions })=0
\end{gathered}
$$

With use of the gauge condition $f_{a}{ }^{\mu \nu \cdots} \partial_{\mu} S=0$ we see that all the terms but the first in these two equations are zero identically. Thus the field equations reduce in this limit to the particularly simple form

$$
\begin{gathered}
(\partial S)^{2}=0 \\
\partial_{\mu}\left(e^{-\xi \kappa \phi} \partial^{\mu} S\right)=0
\end{gathered}
$$


These two equations constrain the classical action of the wave train. With their help we will derive the action for the motion of the wave packet, and compute the dilaton force acting on it. We note that the first of the two equations is nothing else but the classical relativistic Hamilton-Jacobi equation describing motion of free massless particles. This is almost exactly what one should expect to be the case, considering that the particles are associated with fields which are massless due to their gauge symmetry. We say almost, since we do expect the dilaton to influence the motion, so it is not that of free particles. To consider this, we recall that if we define the vector field $V_{\mu}$ along the trajectory $\Gamma$ of a particle according to

$$
V_{\mu}=\left.\partial_{\mu} S\right|_{\Gamma}
$$

(where this definition is always possible at least locally) we can rewrite the total action as the line integral

$$
S=\int_{\Gamma} V_{\mu} d x^{\mu}
$$

after eliminating the first derivatives with help of the equations of motion. The simple substitution $d x^{\mu}=\dot{x}^{\mu} d \lambda$ valid along the trajectory $\Gamma$ where $\lambda$ is the parameterization of $\Gamma$, enables us to rewrite the action above as

$$
S=\int_{\Gamma} V_{\mu} \dot{x}^{\mu} d \lambda
$$

The overdot here denotes the derivative with respect to the parameter $\lambda$. Then the constraints Eq. (42) can be rewritten as

$$
\begin{gathered}
V_{\mu} V^{\mu}=0 \\
\partial_{\mu}\left(e^{-\xi \kappa \phi} V^{\mu}\right)=0
\end{gathered}
$$

As we will now show, these three identities suffice to recover the expression for the classical action. The two constraints have to be treated on a different footing, though. As can be seen from a simple one dimensional example, the second constraint, if inserted in the action with a Lagrange multiplier, 
would give rise to a nonlocal theory. Thus, for consistency with our perturbative approach, as we are disregarding back-reaction, we will only impose it at the level of equations of motion, and not in the Lagrange multiplier method. Therefore, our theory is defined by

$$
\begin{gathered}
S=\int_{\Gamma} d \lambda\left(V_{\mu} \dot{x}^{\mu}+\eta V^{\mu} V_{\mu}\right) \\
V_{\mu} V^{\mu}=0 \\
\partial_{\mu}\left(e^{-\xi \kappa \phi} V^{\mu}\right)=0
\end{gathered}
$$

where $\eta$ is the Lagrange multiplier. The second of these equations is clearly the $\eta$ equation of motion, as can be seen by direct variation. The third equation is effectively what introduces the dilaton. So, varying the action with respect to $V_{\mu}$ gives $V^{\mu}=-\dot{x}^{\mu} / 2 \eta$, and the differential constraint then becomes

$$
\partial_{\mu}\left(e^{-\xi \kappa \phi} \frac{\dot{x}^{\mu}}{\eta}\right)_{\Gamma}=0
$$

from which, after noticing that $\partial_{\mu} \dot{x}^{\nu}=0$, we deduce, with help of $\dot{x}^{\mu} \partial_{\mu}=\frac{d}{d \lambda}$,

$$
\frac{d}{d \lambda}\left(\frac{e^{-\xi \kappa \phi}}{\eta}\right)_{\Gamma}=0
$$

or in other words, $\exp (-\xi \kappa \phi)=C \eta$ along the trajectory $\Gamma$ where $C$ is a (multiplicative) integration constant. Choosing it to be $C=-1 / 4$ we can rewrite the resulting action as

$$
\begin{gathered}
S=\int_{\Gamma} d \lambda e^{\xi \kappa \phi} \dot{x}_{\mu} \dot{x}^{\mu} \\
\dot{x}_{\mu} \dot{x}^{\mu}=0
\end{gathered}
$$

where the second equation is the standard dispersion constraint for massless particles, stating that they move along null lines of the space-time. The standard variational procedure combined with the null condition then yields, after simple algebra,

$$
\frac{d^{2} x^{\mu}}{d \lambda^{2}}=-\xi \kappa \partial_{\nu} \phi \frac{d x^{\nu}}{d \lambda} \frac{d x^{\mu}}{d \lambda}
$$


At this point we invoke the Principle of Equivalence to extend this equation to a curved geometry. We assume that it is to be applied in the Einstein conformal reference frame, as would be the most natural way for its extension. Then, the equation of motion becomes

$$
\frac{d^{2} x^{\mu}}{d \lambda^{2}}+\Gamma_{\nu \lambda}^{\mu} \frac{d x^{\nu}}{d \lambda} \frac{d x^{\lambda}}{d \lambda}=-\xi \kappa \partial_{\nu} \phi \frac{d x^{\nu}}{d \lambda} \frac{d x^{\mu}}{d \lambda}
$$

It is illustrative to compare this equation with the ansätz of [14. We recall that the field theory defined by Eqs. (37-38) was derived from the Maxwell-like Lagrangian for the $p$-form field strength:

$$
S_{M}=-\frac{1}{p !} \int d^{4} x \sqrt{g} e^{-\xi \kappa \phi} F^{\mu \nu \lambda \ldots} F_{\mu \nu \lambda \ldots}
$$

Inspection of this Lagrangian shows that a metric field redefinition (conformal transformation)

$$
\bar{g}_{\mu \nu}=e^{-\alpha \xi \kappa \phi} g_{\mu \nu}
$$

with $\alpha=1 /(2-p)(\alpha=2 /(D-2 p)$ in $D$ dimensions $)$ removes any explicit reference to the dilaton in the new frame and renders the Lagrangian canonical,

$$
S_{M}=-\frac{1}{p !} \int d^{4} x \sqrt{\bar{g}} \bar{F}^{\mu \nu \lambda \ldots} \bar{F}_{\mu \nu \lambda \ldots}
$$

where the overbar refers to the use of the conformally rescaled metric as the only field to change. Of course, the above is true provided $p \neq 2(p \neq D / 2$ in $D$ dimensions $)$, since the Maxwell Lagrangian density for this specific ratio is a conformal invariant. If we applied this same conformal transformation to the connexion of Eq. (52) we would obtain after substitution of the new metric

$$
\frac{d^{2} x^{\mu}}{d \lambda^{2}}+\bar{\Gamma}_{\nu \lambda}^{\mu} \frac{d x^{\nu}}{d \lambda} \frac{d x^{\lambda}}{d \lambda}=0
$$

in perfect agreement with the ansätz of [14] for $p \neq 2(p \neq D / 2)$ ! However, our argument would equally well apply to the $p=2(p=D / 2)$-form field strength, as it is derived without reference to conformal properties of the associated Lagrangian. Therefore, as a consequence, the photon's 
motion is also along the trajectories described by Eq. (52) with the appropriate conformal coupling $\xi$.

Yet the dilaton force appearing explicitly in Eq. (52) does not produce any deviations in the geometry of motion as described in the absence of the dilaton. It is, indeed, the aforementioned property of null geodesics that they do not change under conformal transformations which preserves the geometry. Namely, if we locally rescale the parameter $\lambda$ according to

$$
\frac{d \bar{\lambda}}{d \lambda}=e^{\Omega(\lambda)}
$$

with

$$
\Omega(\lambda)=-\left.\xi \kappa \phi(x)\right|_{\Gamma}
$$

and note that the null condition is invariant under this redefinition,

$$
g_{\mu \nu} \frac{d x^{\mu}}{d \lambda} \frac{d x^{\nu}}{d \lambda}=e^{2 \Omega} g_{\mu \nu} \frac{d x^{\mu}}{d \bar{\lambda}} \frac{d x^{\nu}}{d \bar{\lambda}}=0
$$

we can rewrite Eq. (52) as the null geodesic equation in terms of the new parameter:

$$
\begin{gathered}
\frac{d^{2} x^{\mu}}{d \bar{\lambda}^{2}}+\Gamma_{\nu \lambda}^{\mu} \frac{d x^{\nu}}{d \bar{\lambda}} \frac{d x^{\lambda}}{d \bar{\lambda}}=0 \\
g_{\mu \nu} \frac{d x^{\mu}}{d \bar{\lambda}} \frac{d x^{\nu}}{d \bar{\lambda}}=0
\end{gathered}
$$

Here all explicit reference to the dilaton has been absorbed away, and hence we can conclude that the dilaton force does not affect the geometry of motion locally. We recall, however, that when the dilaton develops singular behavior, such as near the black hole singularity, the transformation (58) may be ill defined there. Thence, it can happen that different observers perceive the singularity differently, according to their proper measure of length. This point has been well illustrated by [14] in their discussion of proper motion of axions and dilatons in the dilaton black hole background of the truncated theory. As a corollary we deduce that the spherically symmetric background with the dilaton can not be distinguished from the dilaton-less case on the basis of geometric aberrations, 
and hence both backgrounds would appear the same in experiments such as the standard tests of General Relativity.

It should be obvious, though, that the dilaton force does produce observable physical effects in a locally inertial frame, although the geometry is independent of it. We offer the following simple illustration of this claim. Consider the Schwarzschild background to $O\left(\alpha^{\prime}\right)$ with the dilaton and radial motion in it. The action describing dynamics of the probe is, in the Einstein frame,

$$
S=\int_{\Gamma} d \lambda e^{\xi \kappa \phi}\left(\frac{1}{1-(2 G M / r)}\left(\frac{d r}{d \lambda}\right)^{2}-\left(1-\frac{2 G M}{r}\right)\left(\frac{d t}{d \lambda}\right)^{2}\right)
$$

supplemented with the null condition. The four-velocity of the probe is

$$
\frac{d x^{\mu}}{d \lambda}=u^{\mu}=\left(\begin{array}{c}
\frac{E}{1-(2 G M / r)} e^{-\xi \kappa \phi} \\
E e^{-\xi \kappa \phi} \\
0 \\
0
\end{array}\right)
$$

where $E$ is the energy integral of the probe. The stationary observer at the distance $r$ from the origin, whose space-time evolution is described by the four-velocity

$$
v^{\mu}=\left(\begin{array}{c}
\frac{1}{\sqrt{1-(2 G M / r)}} \\
0 \\
0 \\
0
\end{array}\right)
$$

detects the energy of the probe in the gravitational-dilatonic well as

$$
h \nu=v_{\mu} u^{\mu}=\frac{E}{\sqrt{1-(2 G M / r)}} e^{-\xi \kappa \phi}
$$

Hence, the redshift as seen by an observer stationary in the Einstein frame will clearly be affected by the dilaton. Therefore, the dilaton indeed does produce observable effects. In passing, we note that the redshift formula, as dictated by the above,

$$
\frac{\nu_{1}}{\nu_{2}}=Z_{G R} e^{\xi \kappa(\phi(2)-\phi(1))}
$$


where $Z_{G R}$ is the redshift in standard GR, is not invariant under conformal transformation. This is a consequence of the fact that observers normally move along time-like geodesics, which are affected by confomal transformations. Thus, the formula above is clearly observer-dependent. This results in the need for a careful definition of local physics (energy-momentum relationships) in presence of the dilaton hair. For example, although the geometric picture obtained with our approach coincides with that of [14, the formula for the redshift which one might attempt to obtain using the expressions for the conformal transform of the metric where the dilaton force is absent would differ from ours, since it would not correspond to the Einstein stationary observer, but to its conformal transform. Numerically, the correction to the GR redshift for light emitted by the Sun is $1+O\left(1 / G M_{\odot} R_{\odot}\right)$ and so amounts to a correction of one part in $10^{79}$.

In a more general situation, when the black hole is charged, the dilaton force would not just affect the dispersion relations, but would also affect the Lorentz force acting on charged particles. However, we will not discuss this case in detail, as the effects in question would be incredibly small, in the absence of a highly charged (i.e. almost critical) source as may be seen from our numerical results of the previous section.

In the remainder of this section we undertake a brief discussion of the other class of induced forces specific to the superstring models of gravity. These are the effects induced by the presence of the Kalb-Ramond axion. One such effect, the rotation of the photon polarization vector in a background with axion hair, has been pointed out previously [6, 25]. Again we will simplify our gedanken experiment to the maximum possible, so we can discuss effects of the axion hair alone, and not as coupled to the dilaton hair. Hence we will assume absence of the dilaton from the theory altogether, presuming that it had decoupled dynamically by a symmetry breaking type mechanism which provided it with a large mass. Such a theory has been noted to posses secondary axion hair on black holes, and more generally, on all rotating, or dyonic matter sources. Therefore, it could be possible, in principle, to conduct experiments testing hair without explicit reference to black 
holes. For the sake of simplicity, we will take this possibility here. We will assume that the axion hair arises from a nonvanishing dyon anomaly source in a flat Minkowski background. In this way we will avoid the unnecessary complication of having to worry about the non-trivial metric in our equations. We expect that our results will be qualitatively unchanged in a curved background, in view of the fact that we assume the validity of the Principle of Equivalence in the Einstein conformal frame.

Our intention is to study the influence of the axion hair on the motion of photons. For this purpose, we can concentrate solely on the (modified) Maxwell's equations describing the photon, as we disregard the backreaction assuming that the contribution of the photon to the action is miniscule compared to the background fields. The linearity of Maxwell's equations with the anomalous axion coupling is consistent with this treatment, as it splits the photon from the background electromagnetic field. We note that the background field and the axion hair are given by the dyon solution of Ref. [0, 8]. Then, we will assume that the observations are being made far away from the dyon. Far away from the dyon, the variation of the gradient of the axion field is negligible compared to the short distance physics of the experiment. Therefore, we can replace $\partial_{\lambda} a=q_{\lambda}=\left(0,0,0, a^{\prime}\right)$ where $q_{\lambda} \approx$ const and we have oriented the local coordinate system so that the gradient is along the vertical axis. The independent equations of motion for the photon are

$$
\partial_{\mu} F^{\mu \nu}-\frac{\sqrt{2}}{2} \kappa \epsilon^{\nu \lambda \sigma \rho} q_{\lambda} F_{\sigma \rho}=0
$$

with $F_{\mu \nu}=\partial_{\mu} A_{\nu}-\partial_{\nu} A_{\mu}$, and the gauge condition $\partial_{\mu} A^{\mu}=0$. Note that here we employ the metric $\eta=(1,-\overrightarrow{1})$ of signature -2 more customary to particle physics, to render the results more transparent. Normally, in the absence of the anomalous coupling, the residual gauge invariance under gauge transformations which solve the free Klein-Gordon equation, allows us to set $A_{0}=0$ and hence employ the Coulomb gauge. However this is not possible here, as due to the anomaly the Coulomb gauge is not unitary and the associated ghosts would not decouple. This can be seen as follows. If we gauge transform $A^{\mu}$ to $A^{\prime \mu}=A^{\mu}+\partial^{\mu} \tilde{\Lambda}$ such that $A^{\prime 0}=0$, the gauge transformation 
$\tilde{\Lambda}$ would have to satisfy the two equations

$$
\begin{aligned}
& \dot{\tilde{\Lambda}}=-A^{0} \\
& \partial^{2} \tilde{\Lambda}=0
\end{aligned}
$$

The first equation is solved by

$$
\tilde{\Lambda}(t, \vec{x})=-\int_{t_{0}}^{t} d \tau A^{0}(\tau, \vec{x})+f(\vec{x})
$$

where $f(\vec{x})$ is some function independent of time to be determined by the boundary conditions. Application of the D'Alembertian to the solution yields

$$
\partial^{2} \tilde{\Lambda}(t, \vec{x})=-\dot{A}^{0}(t, \vec{x})+\int_{t_{0}}^{t} d \tau \vec{\nabla}^{2} A^{0}(\tau, \vec{x})+\vec{\nabla}^{2} f(\vec{x})
$$

where $\vec{\nabla}^{2}$ is the spatial Laplacian. Using the equations of motion for $A^{0}$, after some tedious algebra Eq. (69) can be rewritten as

$$
\partial^{2} \tilde{\Lambda}(t, \vec{x})=-\dot{A}^{0}\left(t_{0}, x\right)+\vec{\nabla}^{2} f(\vec{x})+\sqrt{2} \kappa \vec{q} \cdot \int_{t_{0}}^{t} d \tau \vec{\nabla} \times \vec{A}(\tau, \vec{x})
$$

Recalling that $\overrightarrow{A^{\prime}}=\vec{A}-\vec{\nabla} \tilde{\Lambda}$, we note that $\vec{A}$ in the integrand above can be substituted with $\overrightarrow{A^{\prime}}$. Defining $\chi=\partial^{2} \tilde{\Lambda}$ we see that

$$
\dot{\chi}=\sqrt{2} \kappa \vec{q} \cdot \vec{\nabla} \times \vec{A}^{\prime}
$$

which in general does not vanish. Hence $\partial^{2} \tilde{\Lambda}$ is generally not constant in time and $\tilde{\Lambda}$ will not represent a legitimate gauge transformation. In other words, there will have to be additional terms included in the equations of motion accounting for the variation of $\tilde{\Lambda}$, which from the analogy with the BRST method will correspond exactly to the gauge field - ghost couplings. In the special case when $\vec{q} \cdot \vec{\nabla} \times \overrightarrow{A^{\prime}}=0$, the Coulomb gauge will be admissible. Then $\vec{q} \perp \vec{\nabla} \times \overrightarrow{A^{\prime}}$, since $\vec{\nabla} \times \overrightarrow{A^{\prime}}=0$ together with the Coulomb gauge condition $\vec{\nabla} \vec{A}^{\prime}=0$ uniquely sets $\partial_{k} \overrightarrow{A^{\prime}}=0$. But this corresponds to a homogeneous background electric field which can be removed by boundary conditions at infinity. 
We note in passing that in the treatment of [26] it was assumed that the vector $q_{\mu}$ is timelike. Thus in its rest frame $\vec{q}=0$ and the last term in Eq. $(70)$ is absent. Therefore the Coulomb gauge is unitary and does belong to the Lorenz class. This is the reason why those authors were able to use it. We remark, however, that at least due to the axion hair it is more natural to expect that the vector $q_{\mu}$ will be spacelike, as above.

We look for the eigensolutions of the Eq. (66), i.e. the possible physical polarization states of the photon in such background. The residual gauge invariance in combination with the fact that we are already working with field configurations belonging to the Lorenz class tells us that there should be only two independent polarization states for each direction of motion. However, as we noted in the previous paragraph, they are not transverse in general. They could be found in two different ways. Either one could find another gauge condition which intersects non-trivially the Lorenz class and propagates freely and then find all polarization states satisfying those two constraints, or one could find the most general solution from the Lorenz class and then gauge away the pure gauge contributions with help of the residual gauge invariance. We adopt the second approach, or in other words, we choose to work with the Fermi Lagrangian:

$$
S=\int d^{4} x\left(-\frac{1}{4} F_{\mu \nu} F^{\mu \nu}-\frac{1}{2 \zeta}\left(\partial_{\mu} A^{\mu}\right)^{2}+\frac{\sqrt{2} \kappa}{4} \epsilon^{\mu \nu \lambda \sigma} q_{\mu} A_{\nu} F_{\lambda \sigma}\right)
$$

The Lagrangian which reproduces the Eq. (66) with the Lorenz gauge condition is given by $\zeta=1$. The equations of motion are

$$
\begin{gathered}
\partial^{2} A^{\mu}-\left(1-\frac{1}{\zeta}\right) \partial^{\mu} \partial \cdot A-\sqrt{2} \kappa \epsilon^{\mu \nu \lambda \sigma} q_{\nu} \partial_{\lambda} A_{\sigma}=0 \\
\partial^{2} \partial_{\mu} A^{\mu}=0
\end{gathered}
$$

Hence the Lorenz gauge constraint is a free field and its value is completely determined by the boundary conditions. The evolution operator of the electromagnetic field is

$$
K^{\mu \nu}=\eta^{\mu \nu} \partial^{2}-\left(1-\frac{1}{\zeta}\right) \partial^{\mu} \partial^{\nu}-\sqrt{2} \kappa \epsilon^{\mu \nu \lambda \sigma} q_{\lambda} \partial_{\sigma}
$$


and therefore the propagator in the momentum picture is

$$
\begin{aligned}
G_{\mu \nu}= & -\frac{\mathrm{i}}{p^{2}+2 \kappa^{2} q^{2}-2 \kappa^{2} \frac{(q \cdot p)^{2}}{p^{2}}+\mathrm{i} \epsilon}\left(\eta_{\mu \nu}+\left(2 \kappa^{2} \frac{(q \cdot p)^{2}}{\left(p^{2}+\mathrm{i} \epsilon\right)^{2}}-1\right) \frac{p_{\mu} p_{\nu}}{p^{2}+\mathrm{i} \epsilon}\right. \\
& \left.+2 \kappa^{2} \frac{q_{\mu} q_{\nu}}{p^{2}+\mathrm{i} \epsilon}-2 \kappa^{2} \frac{q \cdot p}{\left(p^{2}+\mathrm{i} \epsilon\right)^{2}}\left(q_{\mu} p_{\nu}+p_{\mu} q_{\nu}\right)+\sqrt{2} \mathrm{i} \kappa \epsilon_{\mu \nu \lambda \sigma} \frac{q^{\lambda} p^{\sigma}}{p^{2}+\mathrm{i} \epsilon}\right)-\mathrm{i} \zeta \frac{p_{\mu} p_{\nu}}{p^{2}+\mathrm{i} \epsilon}
\end{aligned}
$$

Since for a spacelike vector $q_{\mu}, m^{2}=-2 \kappa^{2} q^{2} \geq 0$, the physical poles of the propagator are determined by the roots of $p^{4}-m^{2} p^{2}-2 \kappa(q \cdot p)^{2}=0$. This equation states that in addition to massless modes there will appear massive modes in the photon spectrum. The parameter $m^{2}$ will measure the photon mass. In general, the mass will be polarization-dependent, and will have to be obtained from diagonalization of an operator matrix much like an of inertia tensor. We will illustrate this in the special case of a photon moving in the tangent plane, orthogonally to $\vec{q}$.

In the Feynman gauge $\zeta=1$ and with boundary conditions s.t. $\partial \cdot A=0$, we can rewrite the Fourier modes of Eq. (66) as

$$
\begin{aligned}
& \ddot{\phi}+\vec{p}^{2} \phi-\sqrt{2} \mathrm{i} \kappa \vec{q} \cdot(\vec{p} \times \vec{A})=0 \\
& \ddot{\vec{A}}+\vec{p}^{2} \vec{A}-\sqrt{2} \kappa \vec{q} \times \dot{\vec{A}}-\sqrt{2} \mathrm{i} \kappa \vec{q} \times \vec{p} \phi=0
\end{aligned}
$$

where overdot denotes derivative with respect to time, and the solution is of the form $A^{\mu}=$ $(\phi(t), \vec{A}(t)) \exp (\mathrm{i} \vec{p} \cdot \vec{x})$. The Lorenz gauge condition reads $\dot{\phi}+\mathrm{i} \vec{p} \cdot \vec{A}=0$. With its help we obtain, after taking its time derivative, and using the $\phi$ equation of motion,

$$
\phi=\frac{\mathrm{i}}{\vec{p}^{2}}(\sqrt{2} \kappa \vec{q} \cdot(\vec{p} \times \vec{A})+\vec{p} \cdot \dot{\vec{A}})
$$

and

$$
\ddot{\vec{A}}-\sqrt{2} \kappa\left(\vec{q} \times \dot{\vec{A}}-\frac{\vec{q} \times \vec{p}}{\vec{p}^{2}}(\vec{p} \cdot \dot{\vec{A}})\right)+\vec{p}^{2} \vec{A}+2 \kappa^{2} \frac{\vec{q} \times \vec{p}}{\vec{p}^{2}}(\vec{q} \cdot(\vec{p} \times \vec{A}))=0
$$

The equation of motion for $\vec{A}$ can be rewritten in the matrix form

$$
\ddot{A}^{j}+M^{j k} \dot{A}^{k}+N^{j k} A^{k}=0
$$


where in the coordinate frame $q_{\mu}=(0,0,0, q)$ the matrices are

$$
M=-\sqrt{2} \kappa q\left(\begin{array}{ccc}
\frac{p_{1} p_{2}}{\vec{p}^{2}} & \frac{p_{2}^{2}}{\vec{p}^{2}}-1 & \frac{p_{2} p_{3}}{\vec{p}^{2}} \\
1-\frac{p_{1}^{2}}{\vec{p}^{2}} & -\frac{p_{1} p_{2}}{\vec{p}^{2}} & -\frac{p_{1} p_{3}}{\vec{p}^{2}} \\
0 & 0 & 0
\end{array}\right)
$$

and

$$
N=\left(\begin{array}{ccc}
\vec{p}^{2}+2 \kappa^{2} q^{2} \frac{p_{2}^{2}}{\vec{p}^{2}} & -2 \kappa^{2} q^{2} \frac{p_{1} p_{2}}{\vec{p}^{2}} & 0 \\
-2 \kappa^{2} q^{2} \frac{p_{1} p_{2}}{\vec{p}^{2}} & \vec{p}^{2}+2 \kappa^{2} q^{2} \frac{p_{1}^{2}}{\vec{p}^{2}} & 0 \\
0 & 0 & 0
\end{array}\right)
$$

Solving the system is a standard exercise in simultaneous diagonalization of two quadratic forms. The situation here is similar to the case of small oscillations with dissipation. This problem in general is not tractable; however, here the matrix coefficient of the highest derivative is unity, and hence the system is diagonalizable. Thus, the standard prescription $A^{j}=A_{0}^{j} \exp (-\mathrm{i} \omega t)$ yields the secular equation

$$
\operatorname{det} C(\omega)=0
$$

where $C(\omega)=N-\mathrm{i} \omega M-\omega^{2}$. As mentioned above, $C$ is very much like an inertia tensor, and its null vectors define the independent polarizations for a given direction of motion. For brevity we will not discuss the most general solution of the system above. After all, the system does represent only an approximate dynamical model and for the actual arbitrary motion of the photon the variation of the axion will come into play. Yet, if one conducts an experiment in the lab, the results will indicate an effective mass term for the photon. To illustrate this, consider a photon moving in a locally horizontal direction and orient the coordinate axes so that the $x$ axis points in the direction of motion. Then $p_{2}=p_{3}=0$. The evolution matrix $C(\omega)$ becomes

$$
C(\omega)=\left(\begin{array}{ccc}
\vec{p}^{2}-\omega^{2} & -\mathrm{i} \sqrt{2} \kappa q \omega & 0 \\
0 & \vec{p}^{2}-\omega^{2}+2 \kappa^{2} q^{2} \frac{p_{1}^{2}}{\vec{p}^{2}} & 0 \\
0 & 0 & \vec{p}^{2}-\omega^{2}
\end{array}\right)
$$

and so the secular equation is, recalling $m^{2}=-2 \kappa q_{\mu} q^{\mu}=2 \kappa q^{2}$,

$$
\left(\vec{p}^{2}-\omega^{2}\right)^{2}\left(\vec{p}^{2}-\omega^{2}+m^{2}\right)=0
$$


The eigenfrequencies are $\omega^{2}=\vec{p}^{2}$ (double root) and $\omega^{2}=\vec{p}^{2}+m^{2}$ (single root). The last formula illustrates the identification of $m^{2}$ as the photon mass. The associated polarization vectors can be computed as the null vectors of $C(\omega)$ for the corresponding eigenfrequencies. We will skip the laborious (but straightforward) algebra and just quote the final solution:

$$
\begin{aligned}
\phi & =U_{1} e^{\mathrm{i} p(x-t)}-U_{2} e^{\mathrm{i} p(x+t)}-\mathrm{i} \frac{p}{m} V_{1} e^{\mathrm{i}\left(p x-\omega_{m} t\right)}+\mathrm{i} \frac{p}{m} V_{2} e^{\mathrm{i}\left(p x+\omega_{m} t\right)} \\
\vec{A} & =\left(U_{1} e^{\mathrm{i} p(x-t)}+U_{2} e^{\mathrm{i} p(x+t)}\right) \vec{e}^{1}+\left(W_{1} e^{\mathrm{i} p(x-t)}+W_{2} e^{\mathrm{i} p(x+t)}\right) \vec{e}^{3} \\
& +\left(V_{1} e^{\mathrm{i}\left(p x-\omega_{m} t\right)}-V_{2} e^{\mathrm{i}\left(p x+\omega_{m} t\right)}\right) \vec{e}^{2}-\mathrm{i} \frac{\omega_{m}}{m}\left(V_{1} e^{\mathrm{i}\left(p x-\omega_{m} t\right)}+V_{2} e^{\mathrm{i}\left(p x+\omega_{m} t\right)}\right) \vec{e}^{1}
\end{aligned}
$$

where $\omega_{m}=\sqrt{p^{2}+m^{2}}$. The $U_{1,2}$ terms are pure gauge. To see that, one can apply the gauge transformation $\Lambda=-\frac{\mathrm{i}}{p}\left(U_{1} e^{\mathrm{i} p(x-t)}+U_{2} e^{\mathrm{i} p(x+t)}\right)$ which propagates freely: $\partial^{2} \Lambda=0$, to remove these degrees of freedom. Hence, the general physical solution is

$$
\begin{aligned}
\phi & =-\mathrm{i} \frac{p}{m} V_{1} e^{\mathrm{i}\left(p x-\omega_{m} t\right)}+\mathrm{i} \frac{p}{m} V_{2} e^{\mathrm{i}\left(p x+\omega_{m} t\right)} \\
\vec{A} & =\left(V_{1} e^{\mathrm{i}\left(p x-\omega_{m} t\right)}-V_{2} e^{\mathrm{i}\left(p x+\omega_{m} t\right)}\right) \vec{e}^{2}-\mathrm{i} \frac{\omega_{m}}{m}\left(V_{1} e^{\mathrm{i}\left(p x-\omega_{m} t\right)}+V_{2} e^{\mathrm{i}\left(p x+\omega_{m} t\right)}\right) \vec{e}^{1} \\
& +\left(W_{1} e^{\mathrm{i} p(x-t)}+W_{2} e^{\mathrm{i} p(x+t)}\right) \vec{e}^{3}
\end{aligned}
$$

It is interesting to note that there is a longitudinal component of the vector potential, as carried by the mass. However, the solution still contains only two independent polarizations, which is a manifestation of the (unbroken) gauge invariance of the theory even in the presence of mass. Note that in the limit $m \rightarrow 0$ the longitudinal massive component transmutes into a pure gauge and can be gauged away. The resulting expression would be just the standard formula from ordinary classical EM in Coulomb gauge.

The explicit formula for the mass can be easily obtained by substituting $\vec{q}^{2}=(\vec{\nabla} a)^{2}$ for appropriate axion field hair. In our case, the full $O\left(\alpha^{\prime}\right)$ axion hair is determined by formula (A13). To get the photon mass, we could truncate it and retain only the dyon contribution, containing the cross term between the electric and magnetic charges. However, the order-of-magnitude estimate 
will be served just as well by the contribution due to the LCS source due to the rotation, for an experiment on Earth's surface. Here we present formulas for both cases, and calculate the mass numerically as seen by experiments on Earth, for comparison. The masses are, after we recover the units, and use $A=2 M R^{2} \omega / 5$ for the angular momentum of Earth, where $\omega_{E}$ the angular velocity of rotation,

$$
\begin{aligned}
& m_{1}^{2}=\frac{4 \pi^{2} \hbar^{4}}{c^{4} g^{4}} \frac{N^{2}}{M_{E}^{2} R_{E}^{4}} \\
& m_{2}^{2}=\frac{16 \pi^{2} G \hbar^{3}}{c^{7} g^{4}} \frac{\omega_{E}^{2}}{R_{E}^{2}}
\end{aligned}
$$

where they correspond to the dionic and rotating sources, respectively. The string tension $\alpha^{\prime}$ is given by $\alpha^{\prime}=2 \kappa^{2} / g^{2}$ where $g$ is the unification scale coupling constant with the typical magnitude $g^{2} \sim 0.1$. We have used the Dirac quantization condition $Q_{e} Q_{m}=\hbar c N / 2$ for the dyon, and computed the dipole field mass near the equator, where it would be the largest. Here $N$ would measure the total charge of Earth in the units of electron charge. The two numerical values for the masses on the surface of Earth are $m_{1} \sim 2 \times 10^{-92} \mathrm{MeV}, m_{2} \sim 4 \times 10^{-65} \mathrm{MeV}$, well within the limit $m_{\gamma}<3 \times 10^{-33} \mathrm{MeV}$. The rotation-generated mass is significantly bigger than the dyonic one, and what's even more interesting, does not depend on the mass of the celestial body which supports the axion hair. Actually, for a body of the radial size $10^{3}$ times smaller than Earth, which rotates much faster than it $\left(\omega / \omega_{E} \sim 10^{8}\right)$, the photon mass generated by the axion hair approaches the experimental limit, but remains well within it as it still lags by 21 orders of magnitude. Thus, even the experiments involving compact rapidly rotating objects such as milisecond pulsars would not disclose the mass of the photon above the experimental limit set by the measurements on Earth.

We conclude this discussion by noting the origin of the constraints on the mass. They can be divided in two classes: the geomagnetic and the cosmological. Both were discussed in [26] and would remain much the same here. For our purposes, however, the geomagnetic constraints are more sensible, as our approximation breaks down when experiments involving photons are conducted over 
large distances, since the variation of the axion field can not be neglected. In closing, we would like to stress that the existence of a very small photon mass could be viewed as an argument in favor of existence of axions, or an axion-like phenomenon.

\section{Conclusion}

In this paper we have analyzed physical properties of some of the recently constructed $4 D$ black hole solutions in string theory. We have discussed similarities and differences between some of the exact solutions of the classical effective action, describing dynamics of the model-independent degrees of freedom which appear in the supergravity and superstring theories. Our focus was on the importance of the higher order corrections in the string tension $\alpha^{\prime}$ and higher genus contributions, and we find that in general these terms may render significant departures from the observed classical behavior. We have also demonstrated that in string perturbation theory the solutions are essentially equivalent with the approximate $O\left(\alpha^{\prime}\right)$ solutions we have constructed previously.

Finally, we have discussed observable consequences of the black hole hair. We have found that the hair indeed yields departures from Einstein's General Relativity. In general, there would appear forces derived from the hair which may influence the geometry of motion as perceived by pointlike test particles in such background, as well as induce different energy-momentum relations from standard GR. In particular, we have demonstrated that the dilaton hair results in different redshift relations for probes moving in gravity-dilaton potential wells. Although for the specific case considered the dilaton force does not produce any deviations in the geometry of motion of massless particles (thus being indistinguishable from standard GR by radar echo delay and deflection of light experiments) we have argued for the occurence of such deviations in the case of massive and/or charged probes. In addition, we have addressed the issue of nontrivial axionic hair and have found that in the regime of small fields, its anomalous coupling to the electro-magnetic field could provide 
the photon with an effective topological mass term.

\section{Acknowledgements}

We would like to acknowledge useful conversations with Don Page. The work of BAC and NK

was supported in part by the Natural Sciences and Engineering Research council of Canada. The work of KAO was supported in part by DOE grant DE-AC02-83ER-40105, and by a Presidential Young Investigator Award. BAC and KAO would like to thank the CERN Theory Division for kind hospitality during part of this research. 


\section{Appendix}

Here we collect the various metric, dilaton and axion solutions. We also indicate all of the appropriate field and charge redefinitions as necessary.

The action in [11, 12, 13] is

$$
S=\int d^{4} x \sqrt{g}\left\{\frac{R}{2 \kappa^{2}}-\frac{1}{\kappa^{2}} \partial_{\mu} \phi^{\prime} \partial^{\mu} \phi^{\prime}-\frac{1}{2 \kappa^{2}} e^{-2 \phi^{\prime}} F^{2}\right\}
$$

(we have multiplied the action in [11, 12, 13] by $-1 / 2 \kappa$ ). It dictates the dilaton field redefinition

$$
\phi^{\prime}=\frac{\kappa \phi}{\sqrt{2}}
$$

and the charge in which $F^{\prime 2}$ is expressed in $\left(F^{\prime 2} \propto Q^{\prime 2}\right)$ is

$$
Q^{\prime 2}=\alpha^{\prime} \frac{Q^{2}}{8}
$$

Similarly, the Sen action is written as

$$
S=\int d^{4} x \sqrt{g}\left\{\frac{R}{2 \kappa^{2}}-\frac{1}{24 \kappa^{2}} e^{-2 \tilde{\phi}} \tilde{H}^{2}-\frac{1}{4 \kappa^{2}} \partial_{\mu} \tilde{\phi} \partial^{\mu} \tilde{\phi}-\frac{1}{16 \kappa^{2}} e^{-\tilde{\phi}} \tilde{F}^{2}\right\}
$$

(we have multiplied the Sen action by $1 / 2 \kappa^{2}$ and performed the Weyl rescaling), so that

$$
\begin{gathered}
\tilde{\phi}=2 \phi^{\prime}=\sqrt{2} \kappa \phi \\
\tilde{H}_{\mu \nu \lambda}=12 \kappa H_{\mu \nu \lambda} \\
\tilde{Q}^{2}=Q^{\prime 2}=\alpha^{\prime} \frac{Q^{2}}{8}
\end{gathered}
$$

(note that $\tilde{F} \propto 2 \sqrt{2} \tilde{Q}$ whereas $F, F^{\prime} \propto Q, Q^{\prime}$ ).

The Kerr-Newmann solution can be written as

$$
\begin{aligned}
d s^{2} & =\left(\frac{r^{2}+A^{2} \cos ^{2} \theta-2 G M r+G q^{2}}{r^{2}+A^{2} \cos ^{2} \theta}\right) d t^{2}-\left(\frac{r^{2}+A^{2} \cos ^{2} \theta}{r^{2}+A^{2}-2 G M r+G q^{2}}\right) d r^{2} \\
& -\left(r^{2}+A^{2} \cos ^{2} \theta\right) d \theta^{2}-2 A^{2} \sin ^{2} \theta\left(\frac{2 G M r-G q^{2}}{r^{2}+A^{2} \cos ^{2} \theta}\right) d t d \phi \\
& -\left(\frac{\left(r^{2}+A^{2}\right)^{2}-A^{2} \sin ^{2} \theta\left(r^{2}+A^{2}-2 G M r+G q^{2}\right.}{r^{2}+A^{2} \cos ^{2} \theta}\right) \sin ^{2} \theta d \phi^{2}
\end{aligned}
$$


and

$$
A_{\mu}=\left(\left(\frac{Q_{e} r}{r^{2}+A^{2} \cos ^{2} \theta}\right), 0,0,-\left(\frac{Q_{e} r A \sin ^{2} \theta}{r^{2}+A^{2} \cos ^{2} \theta}\right)\right)
$$

where the charge in the metric is given by $q^{2}=\left(\pi \alpha^{\prime} / \kappa^{2}\right) Q^{2}$ in terms of the charge given in $A_{\mu}$ and hence in $F_{\mu \nu}$. The metric given in [11, 12, 13] is (they did not consider the rotating case)

$$
d s^{2}=\left(1-\frac{2 G M}{\rho}\right) d t^{2}-\left(1-\frac{2 G M}{\rho}\right)^{-1} d \rho^{2}-\rho\left(\rho-\frac{Q^{\prime 2}}{G M}\right) d \Omega
$$

and that of Sen

$$
\begin{aligned}
d s^{2}= & -\frac{\tilde{\rho}^{2}+A^{2} \cos ^{2} \theta-2 m \tilde{\rho}}{\tilde{\rho}^{2}+A^{2} \cos ^{2} \theta+2 m \tilde{\rho} \sinh ^{2} \frac{\alpha}{2}} d t^{2}+\frac{\tilde{\rho}^{2}+A^{2} \cos ^{2} \theta+2 m \tilde{\rho} \sinh ^{2} \frac{\alpha}{2}}{\tilde{\rho}^{2}+A^{2}-2 m \tilde{\rho}} d \tilde{\rho}^{2} \\
& +\left(\tilde{\rho}^{2}+A^{2} \cos ^{2} \theta+2 m \tilde{\rho} \sinh ^{2} \frac{\alpha}{2}\right) d \theta^{2}-\frac{4 m \tilde{\rho} A \cosh ^{2} \frac{\alpha}{2} \sin ^{2} \theta}{\tilde{\rho}^{2}+A^{2} \cos ^{2} \theta+2 m \tilde{\rho} \sinh ^{2} \frac{\alpha}{2}} d t d \phi \\
& +\left(\left(\tilde{\rho}^{2}+A^{2}\right)\left(\tilde{\rho}^{2}+A^{2} \cos ^{2} \theta\right)+2 m \tilde{\rho} A^{2} \sin ^{2} \theta+4 m \tilde{\rho}\left(\tilde{\rho}^{2}+A^{2}\right) \sinh ^{2} \frac{\alpha}{2}+4 m^{2} \tilde{\rho}^{2} \sinh ^{4} \frac{\alpha}{2}\right) \\
& \times \frac{\sin ^{2} \theta}{\tilde{\rho}^{2}+A^{2} \cos ^{2} \theta+2 m \tilde{\rho} \sinh ^{2} \frac{\alpha}{2}} d \phi^{2}
\end{aligned}
$$

where $\sinh ^{2}(\alpha / 2)=\tilde{Q}^{2} /\left(2 G^{2} M^{2}-\tilde{Q}^{2}\right), 2 m=\left(2 G^{2} M^{2}-\tilde{Q}^{2}\right) / G M$.

We turn now to the dilaton and axion solutions; our $O\left(\alpha^{\prime}\right)$ solution is [10], for the dilaton,

$$
\begin{aligned}
\phi(r, \theta) & =\frac{\alpha^{\prime} \sqrt{2}}{16 \kappa}\left(\left[\frac{-1}{G M r}\left[2\left(1+\frac{G M}{r}+\frac{4 G^{2} M^{2}}{3 r^{2}}\right)+\left(Q_{e}^{2}-Q_{m}^{2}\right)\right]\right.\right. \\
& \left.+A^{2}\left[\frac{1}{2 G^{3} M^{3} r}\left(1+\frac{G M}{r}+\frac{8 G^{2} M^{2}}{3 r^{2}}+\frac{6 G^{3} M^{3}}{r^{3}}+\frac{64 G^{4} M^{4}}{5 r^{4}}\right)+\frac{Q_{e}^{2}-Q_{m}^{2}}{3 G M r^{3}}\right]+\ldots\right] P_{0}(\cos \theta) \\
& +\left[\frac{2 Q_{e} Q_{m} A}{G M r^{2}}+\ldots\right] P_{1}(\cos \theta) \\
& \left.+\left[\frac{2 A^{2}}{3 G M r^{3}}\left[\frac{14}{5}\left(1+\frac{3 G M}{r}+\frac{48 G^{2} M^{2}}{7 r^{2}}\right)+\left(Q_{e}^{2}-Q_{m}^{2}\right)\right]+\ldots\right] P_{2}(\cos \theta)+\ldots\right)
\end{aligned}
$$

and the axion

$$
a(r, \theta)=\frac{6 \alpha^{\prime} \sqrt{2}}{8 \kappa}\left(\left[-\frac{Q_{e} Q_{m}}{6 G M r}\left(1-\frac{A^{2}}{3 r^{2}}+\ldots\right)\right] P_{0}(\cos \theta)\right.
$$




$$
\begin{aligned}
& +\left[\frac{A}{12 G M r^{2}}\left[-\frac{5}{2}\left(1+\frac{2 G M}{r}+\frac{18 G^{2} M^{2}}{5 r^{2}}\right)-\left(Q_{e}^{2}-Q_{m}^{2}\right)\right]+\ldots\right] P_{1}(\cos \theta) \\
& \left.+\left[\frac{Q_{e} Q_{m} A^{2}}{9 G M r^{3}}+\ldots\right] P_{2}(\cos \theta)+\ldots\right)
\end{aligned}
$$

where contributions to dilaton from electric and magnetic charges, rotation and Gauss-Bonnet curvature terms have been included. The dilaton solution in [11, [12, [13] for a nonrotating electrically charged black hole is

$$
\phi^{\prime}=\frac{1}{2} \log \left(1-\frac{Q^{\prime 2}}{G M \rho}\right)
$$

and for a rotating eletrically charged black hole 19 is

$$
\tilde{\phi}=-\log \left(\frac{\tilde{\rho}^{2}+\frac{\tilde{Q}^{2}}{G M} \tilde{\rho}+A^{2} \cos ^{2} \theta}{\tilde{\rho}^{2}+A^{2} \cos ^{2} \theta}\right)
$$

When expanded to $O\left(\alpha^{\prime}\right)$, with the coordinate transformations Eq. (12) and conventions of Eq. (A5) to (A7), these two solutions are identical with the purely electric contribution in Eq. (A12).

Now we contrast the axion solutions to ours. In the case of [13] the axion is zero identically since they do not include the LCS source and work with purely electric (or magnetic) configurations. Before we can compare the Sen solution with ours, we need to dualize it first. The axion solution of Sen is expressed in terms of the Kalb-Ramond 2-form

$$
\tilde{B}_{t \phi}=\frac{2 m \tilde{\rho} A \sinh ^{2} \frac{\alpha}{2} \sin ^{2} \theta}{\tilde{\rho}^{2}+A^{2} \cos ^{2} \theta+2 m \tilde{\rho} \sinh ^{2} \frac{\alpha}{2}}
$$

We need to dualize it to the pseudoscalar axion which we wrote the solutions in terms of. This can easily be done with the following formulas. Sen's normalizations are such that

$$
\tilde{H}_{\mu \nu \lambda}=\partial_{\lambda} \tilde{B}_{\mu \nu}-\frac{1}{4} \tilde{A}_{\lambda} \tilde{F}_{\mu \nu}+(\text { cyclic permutations })
$$

where the gauge terms must be included in the Chern-Simons combination since the twisting procedure which Sen employs convolutes the electric charge with the angular momentum producing a non-vanishing gauge anomaly. We have noticed this effect previously, when working in a rotating 
background to order $O\left(\alpha^{\prime}\right)$ [10]. Then from the action, the $\tilde{H}_{\mu \nu \lambda}$ is related to our $H_{\mu \nu \lambda}$ by (A6) or in terms of forms,

$$
\tilde{H}=2 \kappa H
$$

Our conventions for the wedge product are defined by $\alpha \wedge \beta=$ Alt $\alpha \otimes \beta$, whereas Sen uses $\alpha \wedge \beta=\frac{(p+q) !}{p ! q !}$ Alt $\alpha \otimes \beta$, with $p$ and $q$ the ranks of the two forms. In the discussion of the dualization in the introduction, we have defined the pseudoscalar axion as $\partial_{\sigma} a=\sqrt{2} \epsilon_{\mu \nu \rho \sigma} H^{\mu \nu \rho}$, or in form notation,

$$
d \tilde{a}=\frac{\sqrt{2}}{2 \kappa} * \tilde{H}
$$

We skip the laborious algebra leading towards the final answer, and only remark that the inclusion of the gauge Chern-Simons form in the definition proves crucial for integrability of $d \tilde{a}$. The final expression for $\tilde{a}$ is

$$
\tilde{a}=-\frac{\sqrt{2} \alpha^{\prime}}{16 \kappa} \frac{Q_{e}^{2} A}{G M} \frac{\cos \theta}{\tilde{\rho}^{2}+A^{2} \cos \theta}
$$

which to order $O\left(\alpha^{\prime}, A\right)$ is identical with our solution. 


\section{References}

[1] M. Green, J. Schwarz, and E. Witten, Superstring Theory, Cambridge University Press (1987); D. Lust and S. Theisen, Lectures on String Theory Springer-Verlag (1990).

[2] C. Lovelace, Phys. Lett. B135 (1984) 75; E.S. Fradkin and A.A. Tseytlin, Phys. Lett. B158 (1985) 316; C.G. Callan, D. Freidan, E.J. Martinec, and M.J. Perry, Nucl. Phys. B262 (1985) 593; A. Sen, Phys. Rev. D32 (1985) 2102; Phys. Rev. Lett. 55 (1985) 1846; C.G. Callan, I.R. Klebanov, and M.J. Perry, Nucl. Phys. B278 (1986) 78; S. Deser and N. Redlich, Phys. Lett. B176 (1986) 350; D. Luest, S. Theisen and G. Zoupanos, Nucl. Phys. B296 (1988) 800; J. Lauer, D. Luest, and S. Theisen, Nucl. Phys. B304 (1988) 236.

[3] D. Gross and J. Sloan, Nucl. Phys. B291 (1987) 41.

[4] C. Callan, R. Myers and M. Perry, Nucl. Phys. B251 (1989) 34.

[5] B.A. Campbell, M.J. Duncan, N. Kaloper and K.A. Olive, Nucl. Phys. B351 (1991) 778.

[6] B.A. Campbell, M.J. Duncan, N. Kaloper and K.A. Olive, Phys. Lett. B251 (1990) 34.

[7] B.A. Campbell, N. Kaloper and K.A. Olive, Phys. Lett. B263 (1991) 364.

[8] K.-Y. Lee and E. Weinberg, Phys. Rev D44 (1991) 3159.

[9] M. Reuter, Class.Quant.Grav. 9 (1992) 751.

[10] B.A. Campbell, N. Kaloper and K.A. Olive, Phys. Lett. B285 (1992) 199.

[11] G.W. Gibbons, Nucl. Phys. B207 (1982) 337.

[12] G.W. Gibbons and K. Maeda, Nucl. Phys. B298 (1988) 741. 
[13] D. Garfinkle, G. Horowitz and A. Strominger, Phys. Rev. D43 (1991) 3140.

[14] A. Shapere, S. Trivedi and F. Wilczek, Mod. Phys. Lett. A6 (1991) 2677.

[15] J. Preskill, P. Schwarz, A. Shapere, S. Trivedi and F. Wilczek, Mod. Phys. Lett. A6 (1991) 2353.

[16] C. Holzhey and F. Wilczek, Nucl. Phys. B380 (1992) 447.

[17] S. Coleman, J. Preskill and F. Wilczek, Phys. Rev. Lett. 67 (1991) 2677.

[18] S. Coleman, J. Preskill and F. Wilczek, Nucl. Phys. B378 (1992) 1975.

[19] A. Sen, Phys. Rev. Lett 69 (1992) 1006.

[20] R. Kallosh, Phys. Lett. B282 (1992) 80.

[21] R. Kallosh, A. Linde, T. Ortín, A. Peet and A. Van Proyen, Stanford Univ. preprint SU-ITP92-13, May 1992.

[22] R. Kallosh and A. Peet, Stanford Univ. preprint SU-ITP-92-27, Sep. 1992.

[23] R. Kallosh, T. Ortín and A. Peet, Stanford Univ. preprint SU-ITP-92-29, Nov. 1992.

[24] M. Bento and O. Bertolami, Phys. Lett. B228 (1989) 348; Phys. Lett. B220 (1989) 113; Phys. Lett. B218 (1989) 162; I. Jack, D.R.T. Jones and N. Mohammedi, Phys. Lett. B220 (1989) 176.

[25] M.J. Duncan, N. Kaloper and K.A. Olive, Univ. of Minnesota preprint UMN-TH-1102/92, Feb. 1992, in press in Nucl. Phys. B.

[26] S.M. Carrol, G. Fields and R. Jackiw, Phys. Rev. D41 (1990) 1231; S.M. Carrol and G. Fields, Phys. Rev. D43 (1991) 3789 


\section{Figure Captions}

Figure 1: The dilaton a) and axion b) solutions of CKO (solid curve) and GMGHSSTWS (dashed curve) as a function of the radial coordinate $r$ (see text). Parameters chosen are $G M^{2}=1$ and $Q^{\prime 2}=\frac{1}{8}$ with $\alpha^{\prime}=1$. In b) we have taken $A / G M=0.1$

Figure 2: Same as Figure 1 with $G M^{2}=10^{4}$ and $\left(Q^{\prime 2} / G M^{2}\right)=1.25 \times 10^{-3}$, and in b) $A / G M=$ 0.1 .

Figure 3: Same as Figure 1 with $G M^{2}=10^{4}$ and $\left(Q^{\prime 2} / G M^{2}\right)=1.53$, and in b) we have taken $A / G M=0.5$. 